\title{
Dépenses d'Assurances, Primes Encaissées: Une Approche Macroéconomique
}

\author{
par J. François Outreville*
}

\section{Introduction}

La distinction traditionnelle entre l'assurance-vie d'une part et les assurances dommages d'autre part, est celle entre épargne et consommation. L'analyse de la demande d'assurance-vie $^{1}$ s'insère généralement de manière satisfaisante dans le cadre récent des choix de portefeuille des épargnants, mais il n'en est pas de même de l'assurance-dommages, que la théorie moderne du consommateur pousserait à l'insertion dans le cadre général des choix de consommation. D'une manière générale, il semble bien difficile de dissocier la demande d'assurance de la demande de tous les autres biens (J.J. Rosa [1977] p. 41).

G. Katona [1954] a proposé une classification pour l'ensemble des dépenses d'un ménage, entre dépenses courantes et habituelles ("habitual outlays") et dépenses d'opportunité ("outlays of choice"). La distinction dépend largement des habitudes propres à chaque ménage. En d'autres termes, chaque ménage connaît parfaitement la part de son revenu qui sera affectée à des dépenses prénues de consommation ou d'épargne, si cette épargne est de nature contractuelle et à plus ou moins long terme. Ainsi, un plan d'épargne logement à cinq ans, oblige le ménage à prévoir les sorties d'argent nécessaires à ce plan car dans le cas contraire, il perdrait les avantages d'un tel plan. Il en est certainement de même d'un contrat d'assurance-vie puisque pendant les trois premières années, un assuré ne peut pas récupérer les primes versées (la valeur de rachat du contrat étant nulle).

Nous pouvons parler d'un certain degré de compressibilité des dépenses d'un ménage. Comme il existe des dépenses de consommation incompressibles, il existe des engagements d'épargne incompressibles à court-terme. Ainsi, en cas de réduction non-anticipée du revenu, un ménage choisira probablement de réduire ses dépenses d'opportunité afin de pouvoir satisfaire à toutes ses dépenses courantes, y compris ses engagements d'épargne.

* Professeur de Finance et Assurance, Faculté des Sciences de l'Administration, Université Laval. Cet article est extrait d'un Doctorat d'état es Sciences Economiques (Orléans, 1979) “Un modèle macroéconomique du secteur de l'Assurance en France", réalisé sous la direction du professeur J.J. Rosa et avec l'aide d'une bourse de recherche de l'Association Internationale pour l'Etude de l'Economie de l'Assurance.

${ }^{1}$ Dans un souci de classification des principaux modèles explicatifs datant de ces dix dernières années, C. Ferry [1977], a distingué les différents types d'approche susceptibles d'expliquer la demande d'assurance. 
Bien sûr, l'acquisition d'un nouveau contrat d'assurance-vie ou le choix d'un crédit sur l'achat d'une nouvelle automobile, ne sont pas des dépenses habituelles, mais dès que les contrats ont été signés, ils le deviennent sans que l'on puisse revenir la plupart du temps sur la décision. D'autre part, en ce qui concerne l'assurance-vie, il est impossible de faire la différence, dans les chiffres, entre les engagements à long terme et les engagements à court terme. Le problème est identique en ce qui concerne l'assurance-dommage. A titre d'exemple, la dépense d'essence pour une automobile à l'occasion de vacances est différente de la dépense d'essence pour cette même automobile pendant l'année. Il en est de même pour la prime d'assurance à caractère provisoire (souscription par exemple à l'occasion de ces mêmes vacances d'une protection temporaire). Cet aspect permanent des dépenses en primes d'assurances, nous incite à penser que ces dépenses seront peu affectées par des mouvements de stock des actifs financiers en possession des ménages, ou par les fluctuations du revenu.

Si l'assurance entraîne une dépense de type "permanente", cette dépense sera une proportion constante dans le temps d'une certaine fraction "permanente" du revenu. Voyons-en les implications au niveau du modèle théorique que nous nous proposons d'utiliser.

\section{Tableau 1}

Classification des dépenses d'un ménage*

\begin{tabular}{|c|c|c|}
\hline & CONSOMMATION & EPARGNE \\
\hline $\begin{array}{c}\text { DEPENSES } \\
\text { COURANTES } \\
\text { (permanentes) }\end{array}$ & $\begin{array}{l}\text { - nourriture - logement } \\
\text { - habillement (la majeure } \\
\text { part) } \\
\text { - impôts } \\
\text { - remboursement de dettes } \\
\text { - primes d'assurance-dom- } \\
\text { mages courantes (incendie, } \\
\text { automobile, responsabilité } \\
\text { civile, etc.) }\end{array}$ & $\begin{array}{l}\text { - caisse de retraite } \\
\text { - plan d'épargne à long } \\
\text { terme } \\
\text { - primes d'assurance-vie } \\
\text { ordinaire }\end{array}$ \\
\hline $\begin{array}{c}\text { DEPENSES } \\
\text { D'OPPORTUNITE }\end{array}$ & $\begin{array}{l}\text { - biens durables } \\
\text { - amélioration de l'habitat } \\
\text { - loisirs } \\
\text { - primes d'assurance-dom- } \\
\text { mages à caractère } \\
\text { provisoire }\end{array}$ & $\begin{array}{l}\text { - acquisitions nouvelles de } \\
\text { titres de placements } \\
\text { liquides (actions, obliga- } \\
\text { tions, dépôts bancaires) } \\
\text { - primes d'assurance-vie à } \\
\text { caractère temporaire }\end{array}$ \\
\hline
\end{tabular}

* Nous avons volontairement exclu de ce tableau les dépenses de santé du fait du caractère obligatoire de la Sécurité Sociale en France. 


\section{Les fondements théoriques}

La première analyse théorique complète de l'assurance basée sur une fonction d'utilité particulière de type $\mathrm{U}(\mathrm{x})=\log \mathrm{x}$, définie par Bernoulli [1738], a été réalisée en France, à propos de l'assurance-incendie par Barrois en 1834.

Menahem Yaari [1964] [1965] a fait l'hypothèse que la demande d'assurance devait être considérée dans le cadre du "processus d'allocation des ressources du consommateur dans le temps".

L'individu est supposé disposer à chaque période de temps, d'un revenu certain $Y_{t}$, $Y_{t+1} \ldots . . Y_{t+T}$, où $t+T$ représente l'âge maximum possible (soit 99 ans pour les calculs actuariels).

Il partage ce revenu certain entre un plan de consommation $\mathrm{C}_{t+i}$ et un plan de patrimoine (de richesse) $W_{t+i}$. A chacun de ces plans est associée une fonction d'utilité $g\left(C_{t+i}\right)$ et $h\left(W_{t+i}\right)$, et l'individu cherche à maximiser son utilité totale $U_{t}$ où

$$
U_{t}=\sum_{i=0}^{T}\left\{a(t+i) \cdot g\left(C_{t+i}\right)+b(t+i) \cdot h\left(W_{t+i}\right)\right\}
$$

où $\mathrm{a}(\mathrm{t}+\mathrm{i})$ et $\mathrm{b}(\mathrm{t}+\mathrm{i})$ sont des fonctions d'actualisation subjective des fonctions d'utilité.

Nous ne considérons volontairement qu'une forme simple de fonction d'utilité, c'est-à-dire que nous excluons les probabilités d'événements.

Le comportement des individus résulte donc de deux niveaux de décisions:

- l'allocation des revenus disponibles entre épargne et consommation,

- l'allocation de la richesse entre les diverses opportunités du marché.

Traiter la demande d'assurance de la même façon que la demande de tout autre bien ou service, soit comme le premier élément de l'optimisation de l'utilité de l'individu, revient à dire que cette demande est fonction du revenu et d'un vecteur de prix $\mathrm{p} 1$ entrant dans la décision de consommation du public. Mais l'assurance, en tant qu'échange d'une somme monétaire actuelle contre une somme monétaire conditionnelle dépendant de la réalisation hypothétique d'un événement, est un actif financier et fait donc intervenir le second élément de l'utilité totale de l'individu. La demande sera donc aussi fonction du patrimoine et d'un vecteur de taux d'intérêts $\mathrm{r}^{\mathrm{k}}$, entrant dans la décision de portefeuille du public.

La dérivation mathématique de la fonction d'utilité que les ménages cherchent à maximiser, a été faite par L.R. Klein ([1961] pp. 192-199).

Si la richesse totale des ménages $\mathrm{W}_{\mathrm{t}+\mathrm{n}}$ est égale à la somme de l'ensemble de ses actifs physiques et financiers $A_{t+n}$ et de son plan d'assurances $Q_{t+n}$

$$
\begin{aligned}
& W_{t+n}=A_{t+n}+Q_{t+n} \quad \text { avec } A_{t+n} \geqslant 0 \quad n=0,1, \ldots \ldots T \\
& \mathrm{Q}_{\mathrm{t}+\mathrm{n}} \geqslant 0 \quad \mathrm{n}=0,1, \ldots . . \mathrm{T}
\end{aligned}
$$


le problème d'allocation du consommateur revient à maximiser sont utilité $U_{t}$ sous une contrainte de richesse positive, et la dérivation mathématique de ce problème nous permet d'exprimer la demande d'assurance suivant une équation de la forme:

$$
Q_{(t)}=Q_{(t)}\left\{Y_{(t)}, A_{(t)}, p^{1}, r^{k}\right\}
$$

Cette même fonction se retrouve dans les travaux de Cummins [1975] et Outreville [1977].

\section{Les hypothèses de comportement}

\subsection{Le patrimoine des ménages}

Pour Fortune ([1973] p. 593, Théorème 4), si l'aversion pour le risque est une fonction décroissante de la richesse, alors un accroissement de la richesse d'un individu induira une baisse de son aversion pour le risque, qui entraînera à son tour une chute de la prime d'assurance. Il vérifie empiriquement cette action négative du patrimoine qui était prédite par son approche théorique. Il s'oppose sur ce point à une série d'études qui mettent en évidence une action positive du patrimoine sur la demande d'assurance-vie.

D'après Fischer ([1973] pp. 136-137), la valeur absolue de la prime est directement proportionnelle à la richesse. Son influence sera positive si l'espérance de rendement du contrat est supérieure au rendement de l'actif sans risque. Il attend donc une influence positive de la richesse que vérifient les estimations d'Anderson et Nevin ([1975] p. 383) et d'Hammond, Houston et Melander [1967] p. 400). Afin de connaître sa quantité optimale d'assurance, le ménage doit connaître la perte maximale encourue par la famille, moins une certaine proportion de sa richesse accumulée.

Ce facteur de proportionalité est une fonction du risque qui caractérise la famille, mais la richesse que le ménage doit prendre en considération est celle représentant uniquement les actifs substituables à un contrat d'assurance. En effet, pour un ménage ayant une aversion pour le risque, si cette aversion s'accroît, sa richesse en actifs substituables diminuera et sa demande d'assurance sera plus grande. De la même manière, si la compagnie d'assurance réduit le coût du contrat d'assurance, la richesse en actifs substituables diminuera au profit de la demande d'assurance. Dans ce cas, et dans ce cas seulement, la richesse en actifs substituables aura une influence négative sur la demande d'assurance. Or si l'on considère l'ensemble du patrimoine des ménages, il est fort probable que son influence soit tout au contraire positive, car la richesse des ménages est fortement corrélée avec le revenu permanent. "La valeur du patrimoine humain et non-humain est égale au revenu permanent escompté du fait du taux de rendement moyen de ce patrimoine" (J. Melitz [1976] p. 24). Il est donc fort probable aussi que, du fait de l'ambiguité due à cette agrégation de toute la richesse, cette variable soit peu significative.

\subsection{Le revenu des assurés}

Alors qu'il existe une ambiguïté à propos de la richesse des ménages, il n'y en a plus à propos du revenu des assurés: celui-ci doit avoir un effet positif. D'ailleurs Fortune [1973] trouve empiriquement une action positive et significative du revenu. Il en est de même dans toutes les autres études. 


\subsection{L'environnement financier}

Conformément à l'hypothèse de l'existence d'actifs substituables à l'assurance, si les taux d'intérêt sur ces actifs augmentent, la demande de ces actifs devrait croître au détriment de la demande d'assurance et l'influence devrait donc être négative.

Notre conclusion s'oppose complètement aux vérifications empiriques faites par Fortune ([1973] p. 597), Klein ([1975] p. 907) ou Jones-Lee ([1975] p. 909), qui mettent en évidence une action positive du taux d'intérêt. Fischer ([1973] p. 137) est le seul qui démontre l'action ambiguë du rendement de l'actif sans risque et Cummins ([1975] p. 71), qui introduit dans on équation le taux de rendement des obligations publiques comme l'alternative à long terme à la demande d'assurance-vie, trouve bien un signe négatif à cette variable.

L'influence du taux d'intérêt sur la demande d'assurance reste donc théoriquement mal définie, mais il nous semble que plutôt que de faire référence à des taux d'actifs financiers que l'on suppose substituables, l'introduction d'un coût d'opportunité entrầnerait un moindre biais. "Le coût d'opportunité de détention d'actifs est égal à la somme du taux de rendement anticipé sur un stock réel quelconque, donc au taux d'intérêt réel anticipé $r_{a}$, plus le taux d'inflation anticipé Pa" (Melitz [1973] p. 24). Il y a quatre-vingts ans que I.Fisher [1907] expliquait la corrélation entre le niveau des prix et le niveau des taux d'interêt (paradoxe de Gibson) par un effet d'anticipation des prix.

\section{Le modèle}

Les sommes assurées totales mesurent la quantité d'assurance demandée sous forme de capital (ou de rente); elles sont les montants des capitaux et des rentes en cours, à une date donnée. Si l'on se réfère à la présentation traditionnelle la plus simple d'une fonction de demande, soit la quantité demandée fonction du prix, cette variable apparaît comme la variable à expliquer par excellence.

Les primes, elles, sont le produit des quantités d'assurance demandées, par le taux de prime, qui est le prix demandé pour un franc d'assurance. Comme nous n'avons aucune connaissance macroéconomique de ce taux de prime, la variable "primes d'assurance" devient la variable par excellence puisqu'elle regroupe les informations sur la quantité et sur le prix d'une part et que d'autre part, c'est la variable qui est retenue par les sociétés d'assurances dans l'établissement de leurs documents comptables. C. Ferry ([1976] p. 59) souligne justement que l'influence du taux de prime amène la principale critique aux travaux jusqu'à présent réalisés, car son introduction comme variable explicative pourrait faire diverger les estimations. Si les auteurs ralliés aux primes d'assurance sont confrontés à la prise en compte du taux de prime, il en est de même de ceux qui analysent la quantité d'assurance demandée. Or aucun test de la demande d'assurance-vie ne considère le taux de prime à l'exception de Fortune ([1973] p. 596) et Mantis-Farmer ([1968] p. 249).

Fortune avance que les variations du taux sont essentiellement définies par des conditions techniques (table de mortalité, réglementation gouvernementale) et que le taux est indépendant des déterminants de la quantité d'assurance demandée. Il intègre dans ses tests une variable "temps" qui doit capter l'influence linéaire du taux de prime sur les sommes assurées. 
Mantis et Farmer intègrent comme variable de prévision, le rapport de l'assurance sur le prix des biens de consommation. C'est cette approche que nous adopterons du fait de son caractère moins aléatoire.

Toutes les autres études impliquent donc que leurs estimations peuvent être biaisées, ou que l'élasticité-prix des quantités demandées est nulle.

En ce qui concerne le coût d'opportunité de détention d'actifs, on fait généralement l'hypothèse que le taux de rendement réel anticipé sur le patrimoine est très stable sur l'ensemble de la période considérée, et l'on utilise le taux d'inflation anticipé (estimé par une moyenne pondérée des taux d'inflation passés) comme mesure du taux nominal $\left(r_{a}+P_{a}\right)$.

Nous avons démontré par ailleurs (Outreville [1979] p. 98) qu'il existait une ambiguïté à utiliser le patrimoine comme variable explicative (cela étant d'autant plus vrai pour un modèle français qu'il n'existe pas de série homogène disponible).

A l'allocation des revenus disponibles entre épargne et consommation, et de la richesse entre les diverses opportunités du marché, nous préférons l'allocation des revenus disponibles entre dépenses permanentes et dépenses d'opportunité.

Le concept de revenu permanent par opposition au revenu transitoire qui est la différence entre le revenu courant et le revenu permanent, a son origine dans la théorie du revenu permanent développée par M. Friedman [1957]. Le concept de revenu normal a lui été développé par ce que l'on pourrait appeler l'Ecole de l'Université de Pennsylvanie, puisque tous les travaux concernant ce concept ont été entrepris à cette université $^{2}$. Mais la différence entre les deux concepts est plus le fait de la terminologie que du contenu ${ }^{3}$.

L'introduction du revenu normal dans la théorie du comportement de l'individu, suppose que l'on admette que le niveau d'actifs de couverture désiré est une fonction du revenu normal. C'est-à-dire que l'on a la relation

(1)

$$
Q^{*}(t)=f\left(\mathrm{YN}_{(\mathrm{t})}^{\mathrm{t}-1}\right)
$$

${ }^{2} L$ 'idée du concept de revenu normal a été introduite par Irving Friend et Robert Jones [1960] puis développée par Paul Taubman [1964] [1965], I. Friend et P. Taubman [1966], I. Friend [1966], J. Crockett et I. Friend [1967].

${ }^{3}$ L'hypothèse de revenu permanent implique un horizon limité à la durée de vie de l'individu, alors que l'hypothèse de revenu normal se réfère au niveau de revenu que l'individu considère comme normal et espéré pour les années à venir. D'autre part, l'hypothèse de revenu permanent implique une corrélation nulle entre revenu transitoire et consommation transitoire alors que l'hypothèse de revenu normal précise seulement que la propension à épargner le revenu transitoire sera plus grande que la propension à épargner le revenu normal.

Dans un monde de parfaite certitude, l'hypothèse de revenu permanent est certainement très réaliste; dans le monde réel une telle hypothèse est peu réaliste comparée à celle de revenu normal. Le comportement du consommateur se comprend mieux si l'on admet que les effets d'un accroissement du revenu, dépendent de la part que l'individu attribue à un accroissement de son revenu normal, ou à un phénomène transitoire.

Statistiquement parlant, le revenu normal est lui calculé comme une moyenne pondérée des revenus passés et présents. 
où $\mathrm{YN}_{(\mathrm{t})}^{\mathrm{t}-1}$ est la valeur du revenu normal espéré à la période $\mathrm{t}-1$, pour la période $\mathrm{t}$.

Une telle relation peut être de forme linéaire, logarithmique ou quadratique. Par souci de simplicité, nous admettons que l'on a une relation linéaire du type:

$$
\mathrm{Q}_{(\mathrm{t})}^{*}=\mathrm{a}+\mathrm{b} \mathrm{YN}_{(\mathrm{t})}^{\mathrm{t}-1}+\mathrm{cF}
$$

où $\mathrm{F}$ est une variable représentant un vecteur de goût (les conditions financières et sociodémographiques propres à chaque individu).

$\mathrm{Au}$ niveau macroéconomique, cette équation concerne les individus qui désirent accroître leur stock d'actifs de couverture d'un montant

(3) $\Delta Q_{(t)}=\alpha\left(Q^{*}(t)-Q_{(t-1)}\right)$

où $\alpha$ est connue comme la variable de vitesse d'ajustement de stock.

Si l'on substitue l'équation (2) dans l'équation (3), on obtient

$$
\Delta \mathrm{Q}_{(\mathrm{t})}=\alpha . \mathrm{a}+\alpha \cdot \mathrm{Y} \mathrm{YN}_{(\mathrm{t})}^{\mathrm{t}-1}+\alpha . \mathrm{F}-\alpha \mathrm{Q}_{(\mathrm{t}-1)}
$$

Un contrat d'assurance entraînant chaque année un montant déterminé de dépenses en primes, le montant d'actifs de couverture "assurance" pour l'année $t$ (c'est-à-dire le montant total des primes pour l'année) sera

$$
\mathrm{Q}_{(\mathrm{t})}=\Delta \mathrm{Q}_{(\mathrm{t})}+\alpha \mathrm{Q}_{(\mathrm{t}-1)}, \alpha \leqslant 1
$$
devient

Si le revenu transitoire est réellement imprévisible pour l'individu, l'équation (3)

(6) $\Delta \mathrm{Q}_{(\mathrm{t})}=\mathrm{d} Y \mathrm{~T}_{(\mathrm{t})}+\alpha\left(\mathrm{Q}^{*}{ }_{(\mathrm{t})}-\mathrm{Q}_{(\mathrm{t}-1)}\right)$

et l'équation (4)

$$
\Delta \mathrm{Q}_{(\mathrm{t})}=\alpha \cdot \mathrm{a}+\alpha \cdot b \mathrm{YN}_{(\mathrm{t})}^{\mathrm{t}-1}+\alpha \cdot \mathrm{c} \mathrm{F}+\mathrm{dYT}(\mathrm{t})-\alpha \mathrm{Q}_{(\mathrm{t}-1)}
$$

Par contre, si le revenu transitoire n'est pas réellement aléatoire, comme c'est le cas en France du fait de la relative stabilité des revenus, l'équation (3) devient alors

$$
\Delta Q_{(t)}=\alpha\left(Q^{*}(t)-d Y T_{(t)}-Q_{(t-1)}\right)
$$

et l'équation (4)

$$
\Delta \mathrm{Q}_{(\mathrm{t})}=\alpha \cdot \mathrm{a}+\alpha \cdot \mathrm{b} \mathrm{YN}_{(\mathrm{t})}^{\mathrm{t}-1}+\alpha \cdot \mathrm{c} \mathrm{F}-\alpha \cdot \mathrm{d} \mathrm{YT}_{(\mathrm{t})}-\alpha \mathrm{Q}_{(\mathrm{t}-1)}
$$

Nous avons fait l'hypothèse au début de cette étude, que l'assurance était une dépense principalement de type "permanent". Nous attendons donc un coefficient positif 
et significatif du revenu normal et par contre un coefficient non significatif pour le revenu transitoire, ce qui nous permettrait de ne pas tenir compte de l'ambiguité théorique que nous venons de décrire ci-dessus. En termes d'élasticité, cela revient à attendre une élasticité du revenu normal $\mathrm{YN}>1$, et une élasticité du revenu transitoire $\mathrm{YT}=0$.

En ce qui concerne la variable $F$, représentative d'un vecteur de goût, nous conserverons l'approche faite dans le premier paragraphe, à savoir une fonction d'un vecteur de prix $\mathrm{p}^{1}$, d'un vecteur de taux d'intérêts $\mathrm{r}^{\mathrm{k}}$ (représenté par le coût d'opportunité $\mathrm{Pa}$ et le taux de rendement réel de l'actif sans risque TANR), et d'un vecteur de variables conjoncturelles et structurelles.

De par sa formulation, le modèle de base de la demande d'assurance tient implicitement compte de l'inflation mais peu du chômage. En effet, du fait des allocations de nonemploi, le revenu annuel des individus reste relativement stable et seules les anticipations d'accroissement de revenu sont affectées. Or la définition du revenu normal peut ne pas répondre entièrement à cette hypothèse.

Il est fort probable que le taux d'accroissement du chômage ait un effet négatif sur la demande de nouveaux contrats, par contre, l'effet d'un niveau élevé de chômage est ambigü; s'il réduit les anticipations futures, il accroît aussi le désir de sécurité des individus et a donc probablement un effet positif sur la demande d'assurance vie.

L'article 3 de la loi de finance de 1967 précise l'exonération partielle des primes d'assurance-vie; une mesure analogue avait déjà été appliquée avec des modalités différentes entre 1950 et 1959 . Une telle mesure a certainement un effet positif sur la demande d'assurance-vie.

Il n'est pas simple de definir l'expression "modifications structurelles" 4 et pourtant sur la période d'étude, un certain nombre d'événements et d'incitations politiques ont marqué le secteur des assurances, entre autres le phénomène de concentration du marché de l'assurance en 1967-68. Malheureusement, ces décisions et leurs conséquences sont souvent mal connues et difficiles à discerner, et une telle étude ne peut être qu'intuitive.

Finalement, afin de mieux tenir compte de l'influence socio-démographique dans le goût des individus, nous introduisons dans notre équation, une variable $\mathrm{N}$ représentant la population, ce qui reviendra à tester la demande par individu sous la forme:

(10) $\frac{Q_{(t)}}{P . N .}=\alpha \cdot a+\alpha \cdot b \frac{Y^{t-1}}{P . N}+\alpha \cdot c \frac{Y_{(t)}}{P \cdot N}+\alpha \cdot d \cdot P a$

$$
+\alpha . e(\text { TANR }-\mathrm{Pa})+\alpha . \mathrm{FISC}+\alpha . \mathrm{g} \cdot \frac{\mathrm{TNPE}}{\mathrm{N}}
$$

\footnotetext{
${ }^{4}$ Nous remercions K. Borch qui nous a suggéré une telle analyse à propos d'un commentaire concernant une étude sur l'agrégation (Outreville [1978]).
} 


$$
\begin{aligned}
\mathrm{Q} & =\text { total des primes de l'exercice par catégories d'assurance } \\
\mathrm{YD} & =\text { revenu disponible courant des ménages } \\
\mathrm{YN} & =\text { revenu normal }\left(=\sum_{\mathrm{i}} \mathrm{w}_{\mathrm{i}} \mathrm{YD}_{\mathrm{t}-\mathrm{i}}, \Sigma \mathrm{w}_{\mathrm{i}}=1\right) \\
\mathrm{YT} & =(\mathrm{YD}-\mathrm{YN}) \text { revenu transitoire } \\
\mathrm{Pa} & =\text { taux d'inflation anticipé } \\
\mathrm{P} & =\text { indice général des prix (déflateur de la PIB) } \\
\mathrm{N} & =\text { population totale } \\
\mathrm{TANR} & =\text { taux de rendement de l'actif sans risque (rente d'état perpétuelle) } \\
\mathrm{FISC} & =\text { effet de l'incitation fiscale (1 entre 1950-1959 et à partir de 1967 } \\
& 0 \text { ailleurs) } \\
\mathrm{TNPE} & =\text { (demandes d'emploi non-satisfaites }- \text { offres d'emploi non-satisfaites) }
\end{aligned}
$$

\section{Les problèmes d'agrégation}

Nous allons voir dans ce paragraphe, qu'en ce qui concerne le secteur des assurances, les relations économiques ne sont pas exemptes d'erreurs de spécification aussi bien du point de vue théorique que du point de vue empirique.

En effet l'analyse macroéconomique permet de définir parfaitement les relations entre agrégats, mais il est bien évident qu'un modèle qui explique un comportement moyen, ne peut en aucun cas expliquer le comportement d'une firme ou d'un ménage individuellement.

\subsection{Agrégation au niveau des ménages}

Bien sûr, il n'y a aucune raison de penser que tous les individus ont le même comportement face au risque et Friedman-Savage [1948] ont même été jusqu'à proposer une fonction d'utilité dont les segments sont concaves ou convexes suivant les classes socio-économiques des individus. Plus récemment, un certain nombre d'études tendent à montrer les limites de la théorie de la maximisation de l'utilité espérée dans les décisions impliquant des situations de risque, du fait de la non-information ou de la mauvaise information dont dispose l'individu ${ }^{5}$. Le besoin de nouveaux modèles de décision a notamment été démontré par Kenneth Arrow en 1973, à son discours de présidence à l'American Economic Association (Arrow [1974] p. 1).

${ }^{5}$ Les principales études empiriques faites à partir d'enquêtes auprès de ménages sont celles de Kunreuther [1976] [1978], et Slovic et al. [1977]. 
S'il existe de telles divergences entre divers groupes d'individus, les résultats empiriques d'une analyse macroéconomique seront sujets à un important biais d'agrégation. Mais, inversement, le fait que la théorie microéconomique sur le comportement de ces mêmes individus ne permette pas de donner une réponse quant à la meilleure spécification possible, cette même analyse macroéconomique sera la meilleure relation possible du comportement "moyen" d'un individu dans un certain environnement économique, toutes choses étant égales par ailleurs, c'est-à-dire les événements socio-économiques (probabilité de risque, valeur de l'information, nature des ménages...) qui affectent les micro-relations, mais que l'on ne peut pas prendre en compte au niveau des macrorelations.

\subsection{Agrégation au niveau des produits de l'assurance}

Il n'y a aucune raison de considérer, d'un point de vue microéconomique, que les comportements dans les différentes lignes de l'assurance-vie sont identiques, et encore moins en ce qui concerne les différents types de contrats de l'assurance-dommage.

La désagrégation dans ce cas peut permettre à l'étude empirique de vérifier le bien fondé de l'analyse. Mais il serait futile de croire qu'il suffit de désagréger pour obtenir de meilleurs résultats:

"Different levels of aggregation require theories with different levels of abstraction" (Grunfeld - Griliches [1960] p. 10).

\subsection{Agrégation au niveau des entreprises du secteur}

L'analyse macroéconomique considère l'ensemble du secteur et uniquement l'ensemble du secteur. Elle ne permet pas de distinguer entre le comportement de petites entreprises et celui de grandes, ni entre celui des entreprises nationalisées, des entreprises du secteur mutualiste ou des sociétés anonymes. Dans une étude précédente (Outreville [1978]) nous montrons qu'en fait l'agrégation au niveau des différents types de firmes semble être une hypothèse raisonnable quel que soit le type d'assurance considéré.

Ces remarques étant faites, il nous reste le confort de penser que ces difficultés affectent tous les modèles macroéconomiques existant à ce jour.

\section{Les résultats empiriques}

\subsection{Résultats de l'approche intégrée}

Chaque équation a été testée par les moindre-carrés simples, en utilisant la procédure de réduction de l'autocorrélation des résidus au premier degré, développée par Cochrane-Orcutt.

Du fait de l'après-guerre et de la nécessité de calculer le revenu normal sur une période de six années pour une moyenne pondérée du revenu courant, notre étude porte sur la période 1953-1976. Nous ne présentons ici que la synthèse des résultats que l'on peut retrouver au complet dans une précédente étude (Outreville [1979]). 
Contrairement à l'ensemble des études sur la demande d'assurance qui ne considèrent que l'assurance-vie, notre approche en termes de dépenses permanentes nous permet d'un point de vue théorique d'envisager la même vérification empirique pour l'ensemble des dépenses d'assurance.

En ce qui concerne l'assurance-vie, l'élasticité du revenu normal est toujours supérieure à 1.37 quelle que soit la branche, et celle du revenu transitoire inférieure à 0.075 . On remarque qu'en ce qui concerne l'assurance collective, le coefficient estimé du revenu transitoire n'est pas significatif; or ce type d'assurance est principalement le fait d'entreprises agissant pour leurs employés et pour des contrats d'assurance-vie temporaire. Par son caractère "obligatoire" et sa nature de "protection pure", cette décision ne peut être évaluée compétitivement par rapport à des actifs substituables sur le marché. D'autre part le signe du coefficient de revenu transitoire est l'inverse de ce qui aurait été espéré si l'on avait considéré la dépense d'assurance comme une épargne d'opportunité. On retrouve le même problème avec le coefficient du coût d'opportunité qui n'est pas significatif dans le cas de l'assurance collective; par contre il est bien de signe négatif comme attendu.

Les résultats empiriques semblent donc bien confirmer, pour l'assurance-vie, les hypothèses que nous avons formulées. De plus, la désagrégation par branche montre quelques variations de comportement, notamment en ce qui concerne l'assurance collective où le coût d'opportunité est bien négatif mais non significatif, mais dans l'ensemble le comportement de chaque branche prise individuellement reste conforme aux résultats de l'agrégat total vie et capitalisation. Le biais d'agrégation dans le cas présent modifiera certainement l'exactitude des estimations mais non par leur signe ou leur degré de signification, ce qui est fondamental.

Si l'on considère l'équation du total des primes dommages agrégées, les résultats sont comparables à l'assurance-vie. La dépense pour ce type d'assurance est bien de type "permanente", alors que le coefficient d'élasticité du revenu transitoire est égal à 0.009 et est non significatif. D'autre part, le signe du taux d'inflation anticipé est bien négatif et significatif. Par contre, le test de Durbin-Watson reste faible même après la correction par la procédure de Cochrane-Orcutt. Cela reflète la moins bonne spécification de cette équation appliquée à l'assurance dommage, ce qui tendrait à confirmer le fait que ce type d'assurance résulte d'un comportement décisionnel différent sur certains points, mais aussi qu'un biais d'agrégation très important subsiste. Entre autre on ne connaît pas dans chaque branche (particulièrement les branches "incendie" et "transports") la part relative des ménages et des contrats industriels.

Si l'ensemble des assurances vie et capitalisation présente un agrégat relativement homogène, il n'est est pas de même de l'assurance dommage. Notre optique macroéconomique, consistant à intégrer dans une même équation l'ensemble de la demande d'assurance, n'est que partiellement vérifiée.

\subsection{L'assurance-vie}

Les résultats concernant l'influence du taux de rendement de l'actif sans risque (TANR), et l'effet de l'incitation fiscale (FISC), ne s'appliquent qu'à l'assurance-vie. Ils sont conformes à ce que l'on espérait. Le taux de rendement réel de l'actif sans risque a 
Tableau III - Elasticités

\begin{tabular}{|c|c|c|c|}
\hline Branche d'assurance & $\begin{array}{l}\text { Revenu } \\
\text { Normal }\end{array}$ & $\begin{array}{c}\text { Revenu } \\
\text { transitoire }\end{array}$ & $\begin{array}{c}\text { Coût } \\
\text { d'opportunité }\end{array}$ \\
\hline TOTAL Vie et Capitalisation & $1.409^{+}$ & $-0.023^{+}$ & $-0.096^{+}$ \\
\hline Capitalisation & $1.437^{+}$ & $-0.021^{+}$ & $-0.281^{+}$ \\
\hline$\&$ populaire & $1375^{+}$ & $-0075^{+}$ & $-0186^{+}$ \\
\hline Collective & $1.548^{+}$ & $-0.023^{-}$ & $-0.024^{-}$ \\
\hline TOTAL Dommages & $1.434^{+}$ & $-0.009^{-}$ & -0.102 \\
\hline Maladie et accidents & $1.617^{+}$ & -0.024 & $-0.080^{-}$ \\
\hline Incendie & $1.364^{+}$ & $+0.008^{-}$ & $+0.012^{-}$ \\
\hline Automobile & 0.744 & $-0.013^{-}$ & $-0.073^{-}$ \\
\hline Transports & $0.670^{+}$ & +0.062 & +0.338 \\
\hline Responsabilité & $1.259^{+}$ & +0.025 & +0.021 \\
\hline $\begin{array}{ll}+ & \text { signifi } \\
- & \text { non-si }\end{array}$ & $\begin{array}{l}\text { de } 1 \% \\
\text { seuil de }\end{array}$ & & \\
\hline
\end{tabular}

bien une action négative et significative sur la demande d'assurance-vie. L'incitation fiscale a bien un effet positif et significatif.

Le niveau de population non-employée accroît la sensibilité au risque des individus, leur besoin de sécurité et donc leur demande d'assurance-vie. Par contre, à court terme, un accroissement du taux de chômage a un effet négatif sur la demande de nouveaux contrats. Ces deux résultats ne sont en rien contradictoire ${ }^{6}$.

\subsection{Analyse des modifications structurelles}

Nous avons essayé de développer une procédure économétrique dont le but principal est de détecter toute modification, structurelle ou non, au cours de la période d'étude. Il va de soi que les résultats d'une telle approche, purement statistique, sont à analyser avec la plus grande circonspection. La plus récente contribution à ce genre d'analyse, est celle de D.J. Poirier [1976], qui étudie les effets linéaires mais aussi non-linéaires de modifications structurelles dans un modèle de régression. Nous limiterons notre analyse empirique au cas le plus simple.

\footnotetext{
${ }^{6}$ Nous remercions L. Eeckhoudt d'avoir récemment confirmé la véracité de notre hypothèse.
} 
Tableau IV

Total Assurances vie et capitalisation

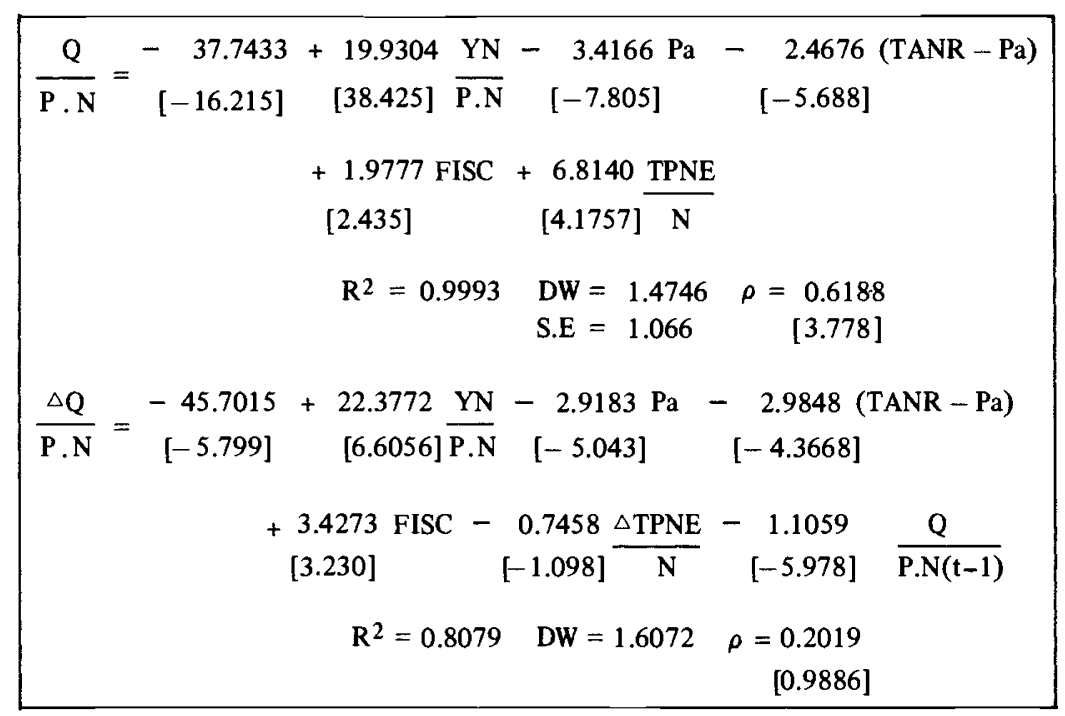

Tableau V

Total Assurances dommage

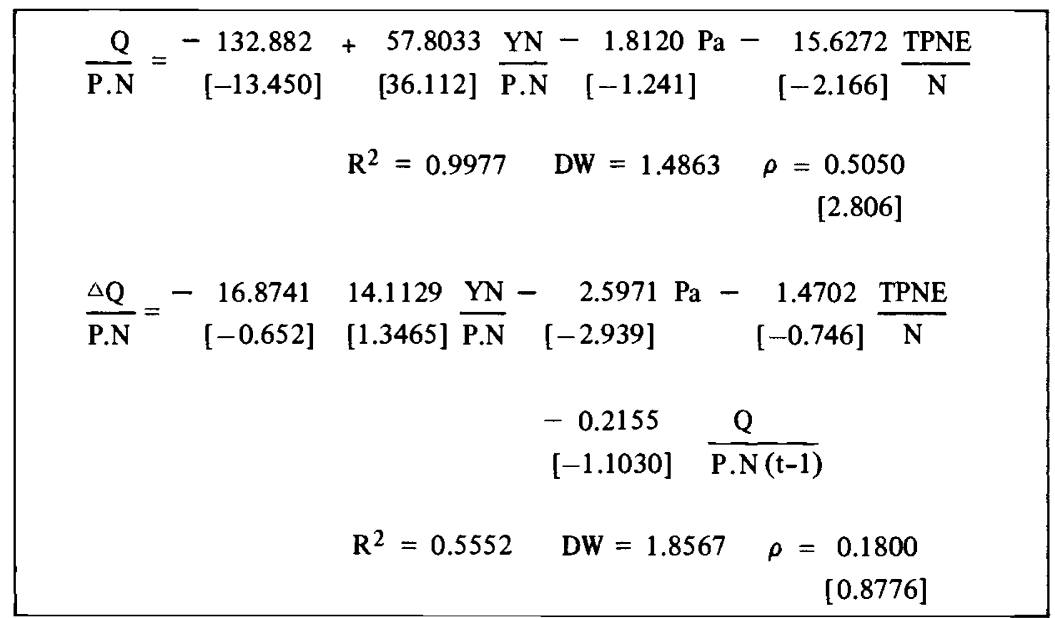

$$
\text { DW }=\text { test de Durbin-Watson }
$$

entre $[$ [ $]=$ test de Student

$\mathrm{SE}=$ écart-type de la régression

$\rho=$ degré d'autocorrélation 
L'idée de cette analyse est de tester, à partir du modèle le plus élaboré pour chaque agrégat, vie et capitalisation d'une part, dommages d'autres part, l'effet de "dummy" variables de niveau, ayant la valeur 0 sur la période $t-j, \ldots . t-1$, puis la valeur 1 sur la période $t, t+1, \ldots . .1976$.

La même équation a été testée en prenant successivement $t=1958,1959 \ldots . .1976$, car il n'était statistiquement pas possible (trop peu de degrés de liberté pour l'estimation des paramètres) de tester le polynôme complet.

L'idée d'une modification structurelle étant d'avoir un impact positif sur la demande, nous ne retenons, pour la présentation des résultats, que la période ou le coefficient de la "dummy" variable est positif. Nous pouvons d'ailleurs préciser que pour toutes les autres années, le coefficient était négatif (sauf en 1959) et toujours non-significatif (sauf en 1974, coefficient significatif mais négatif), et cela pour les deux types d'agrégats. La seule différence entre les assurances vie et capitalisation d'une part, dommage d'autre part, se mesure en 1969, 1970. Nous présentons graphiquement le degré de signification (mesuré par le test de Student) d'une éventuelle modification structurelle affectant la demande à partir de chaque année t entre 1965 et 1972 [Figures 1a) et 1b)].

Figures 1 -Analyse des effets des modifications structurelles sur la demande d'assurance

1a) Assurances vie et capitalisation

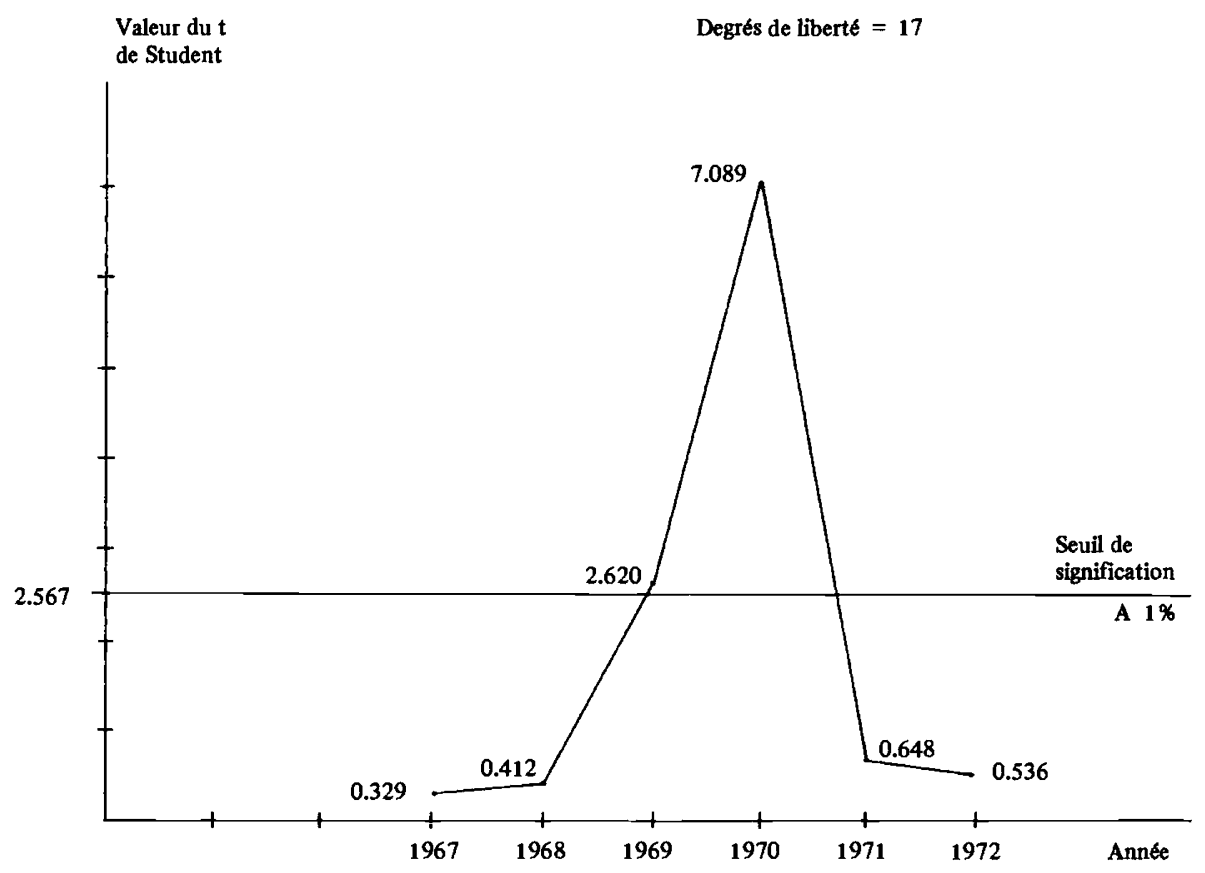


1b) Assurances dommages

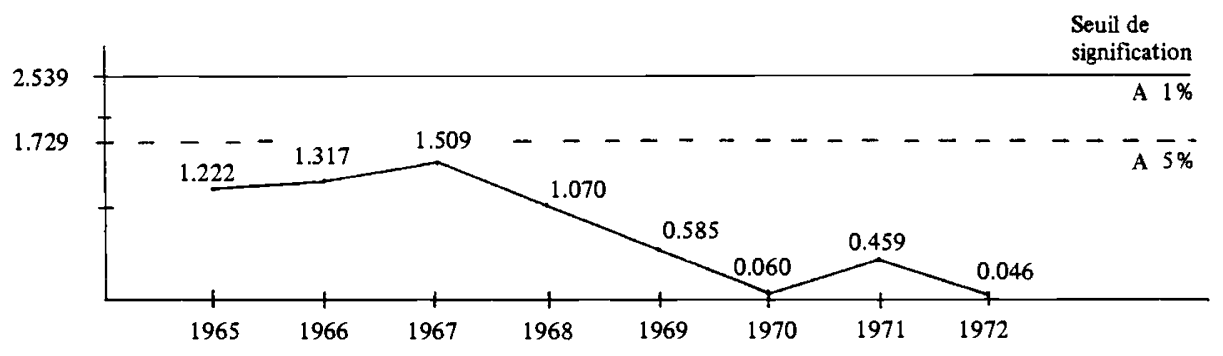

Ainsi, aucune modification structurelle ne semble affecter la demande d'assurance sur la période 1950-1976, à une exception notable près, la demande d'assurance vie et capitalisation à partir de 1969 et surtout 1970 . Or la restructuration du marché de l'assurance vie avec la concentration des entreprises nationalisées est effective en 1968. Un délai de deux ans semble un peu long et nous préférons, faute de plus ample information ne pas conclure sur ce point. Pourtant il s'est bien produit une modification structurelle, et cela uniquement pour la demande d'assurance vie et capitalisation.

\section{Simulation d'hypothèses et effets sur les primes}

La méthode consiste à étudier analytiquement le modèle linéarisé au voisinage d'une trajectoire de référence. Compte tenu de la spécification numérique du modèle et d'hypothèses sur les variables exogènes, on montre quels sont les mécanismes importants.

Afin d'observer les effets des variables exogènes au secteur, nous avons formulé quatre hypothèses de comportement de l'économie dans les années futures:

hypothèse 1: inflation élevée chômage élevé croissance faible

hypothèse 2: inflation réduite chômage élevé croissance faible

hypothèse 3: inflation réduite chômage réduit croissance faible

hypothèse 4: inflation élevée chômage réduit croissance forte

Les deux premières hypothèses sont proches de la réalité actuelle ou envisagée; la dernière hypothèse est l'alternative proposée à la situation résultant des deux premières 
hypothèses. Seule la troisième hypothèse est actuellement peu réaliste, mais elle correspond au désir de tester individuellement l'impact du chômage.

Les graphiques que nous présentons sont les résultats des simulations des hypothèses 1 à 4 pour le total des primes émises d'une part et pour ces mêmes primes en volume par individu (Q/P.N.). L'hypothèse de référence (ou de continuité) (*) est la simple extrapolation dans le futur d'une croissance constante égale à la moyenne observée sur la période 1960-1976.

D'après les résultats de l'analyse en "valeur" (figures 2 et 3) il apparaît nettement qu'à une forte inflation correspond un fort accroissement des encaissements de primes, mais cet impact est beaucoup trop important, c'est pourquoi il est plus intéressant d'analyser l'évolution des primes en "volume" par individu (figures 4 et 5). Dans les deux cas, assurances vie et capitalisation ou assurances dommage, les résultats sont bornés par l'hypothèse de référence compte tenu d'une inflation moyenne ('*') et compte tenu d'une inflation forte (' + '). En cas de forte inflation, les résultats futurs seront meilleurs que ne le laisse supposer une simple extrapolation de la croissance moyenne actuelle. En cas de retour à un taux d'inflation moyen ( $5.75 \%$ par an), les résultats futurs seront par contre inférieurs à ce que donne une simple extrapolation de la croissance moyenne actuelle. L'hypothèse la moins favorable est dans tous les cas la première, la plus favorable dans tous les cas la dernière. Une forte croissance de l'économie semble donc être le moteur principal de la croissance des encaissements de primes pour le secteur des assurances.

La différence entre les hypothèses 2 et 3 est peu significative (elle est inverse du fait de l'effet inverse du chômage). Si l'on observe principalement l'hypothèse 2 , plus réaliste par rapport à la situation économique actuelle, il apparaît qu'elle est beaucoup plus favorable au secteur des assurances vie et capitalisation qu'à celui des assurances dommage où elle s'écarte peu de la première hypothèse.

La prise en compte des commissions et charges pour le calcul des primes pures affecte peu les conclusions précédentes ${ }^{7}$ - (a) en ce qui concerne les assurances vie et capitalisation, l'effet des prix est immédiat et l'élasticité calculée sur la période d'estimation 19561975 très faible: 0.032 - l'effet d'économie d'échelle s'étant avéré non significatif, on peut appliquer sans restrictions les conclusions précédentes aux primes pures; (b) en ce qui concerne les assurances dommage, l'effet des prix est beaucoup plus diffus dans le temps (l'estimation par les lags d'Almon indique 7 années, ce qui nous semble probablement excessif), d'autre part, il existe probablement un effet d'économie d'échelle même faible. Il s'ensuit qu'à un taux d'inflation constant dans le temps, la croissance des primes pures sera égale ou légèrement supérieure à celle des primes commerciales. Par contre, après une période de forte inflation, l'impact sur les commissions et charges se prolongera pendant quelques années et durant ce délai, la croissance des primes pures sera légèrement inférieure à celle des primes commerciales.

\footnotetext{
${ }^{7}$ L'analyse macroéconomique de l'impact des commissions et charges dans les comptes des compagnies d'assurance (Outreville [1979] pp. 294-309) montre que la relation proportionnelle simpliste liant les commissions et charges à la prime pure n'est pas réaliste mais est largement affectée par la conjoncture économique.
} 


\section{Conclusion}

Cette étude a permis de formuler une alternative macroéconomique à l'étude de la demande d'assurance. De par sa nature, une telle approche est généralisable à l'ensemble des intermédiaires financiers bancaires et non-bancaires. Cependant la principale limite à sa validité concerne le problème d'agrégation.

Que peut-on conclure au sujet du lien entre le développement de l'assurance en France, et l'évolution de l'environnement économique ?

Dans la mesure où les variables explicatives du modèle sont directement liées au revenu national, à l'inflation, au chômage et au taux de rendement des actifs financiers, il est difficile de conclure que l'assurance se développe indépendamment de l'évolution économique en général, bien au contraire. Il suffit de reprendre les résultats des simulations pour s'en convaincre.

Bien que la modélisation soit incontestablement complexe et nécessite un affinement, notamment pour mieux cerner la réalité des divers types d'assurance, elle apparaît ainsi comme possible, et de ce fait la prévision à terme n'est pas à exclure.

\section{REFERENCES}

ANDERSON, D. et J.R. NEVIN [1975]: "Determinants of young marrieds' life insurance purchasing behavior", Journal of Risk and Insurance, 42 (septem bre 1975), 375-387.

ARROW, K.J. [1974]: "Limited knowledge and economic analysis", American Economic Review, 64 (mai 1974), 1-10.

BARROIS, T. [1834]: Essai sur l'Application du Calcul des Probabilités aux Assurances contre l'Incendie, Ed. Daniel, Lille.

BERNOULLI, D. [1738]: "Exposition of a new theory on the measurement of risk", Econometrica, 22 (janvier 1954), 23-46. Traduction de "Specimen Teoria Nova de Mensura Sortis", St. Petersbourg, 1738.

CROCKETT, J. et I. FRIEND [1967]: "Consumer investment behavior", in Determinants of Investment Behavior, National Bureau of Economic Research, New York.

CUMMINS, J.D. [1975]: An Econometric Model of the Life Insurance Sector in the U.S. Economy, Lexington Books, Lexington (Mass.).

FERRY, C. [1976]: La Demande d'Assurance: Analyse Théorique et Empirique, Thèse complémentaire, Université de Dijon.

FERRY, C. [1977]: "L'approche empirique de la demande d'assurance-vie", Geneva Papers on Risk and Insurance, 5 (février 1977), 22-34.

FISCHER, S. [1973]: “A life cycle model of life insurance purchasing”, International Economic Review, 14 (février 1973), 132-152.

FISHER, I. [1907]: The Rate of Interest - Its Nature, Determination and Relation to Economic Phenomena, Mac Millan, Londres.

FORTUNE, P. [1973]: “A theory of optimal life insurance: Development and tests", Journal of Finance, 28 (juin 1973), 587-600.

FRIEDMAN, M. [1957]: $A$ Theory of the Consumption Function, Princeton University Press.

FRIEDMAN, M. et L.J. SAVAGE [1948]: "The utility analysis of choices involving risk", Journal of Political Economy, 56 (août 1948), 279-304. 
FRIEND, I. [1966]: “The propensity to save in India", in Economic Development: Issues and Policies, M. Vohra \& Co., Bombay.

FRIEND, I. et R. JONES [1960]: “The concept of saving”, in Conference on Consumption and Saving, Vol. II, Université de Pennsylvanie.

FRIEND, I. et P. TAUBMANN [1966]: "The aggregate propensity to save: Some concepts and their application to international data", Review of Economics and Statistics, 48 (mai 1966), 113-123.

GRUNFELD, Y. et Z. GRILICHES [1960]: “Is aggregation necessarily bad ?" Review of Economics and Statistics, 42 (février 1960), 1-13.

HAMMOND, J.D., D.B. HOUSTON et E.R. MELANDER [1967]: "Determinants of household life insurance premium expenditures: An empirical investigation", Journal of Risk and Insurance, 34 (février 1967), 397-408.

JONES-LEE, M.W. [1975]: “Optimal life insurance: Comment”, Journal of Finance, 30 (juin 1975), 902-903.

KATONA, G. [1954]: "Variability of consumer behavior", in G. Katona (Ed.), Contributions of Survey Methods to Economics, Columbia University Press, New York.

KLEIN, L.R. [1961]: The Keynesian Revolution, Mac Millan, New York.

KLEIN, M. [1975]: “Optimal life insurance: Comment”, Journal of Finance, 30 (juin 1975), 904-908.

KUNREUTHER, H. [1976]: “Limited knowledge and insurance protection”, Public Policy, 24 (printemps 1976), 227-261.

KUNREUTHER, H. [1978]: Disaster Insurance Protection: Public Policy Lessons, John Wiley \& Sons, New York.

MANTIS, G. et R.S. FARMER [1968]: “Demand for life insurance”, Journal of Risk and Insurance, 35 (mars 1968), 247-265.

MELITZ, J. [1976]: “La demande de monnaie en France”, Statistiques et Etudes Financières de I'INSEE, 11.

OUTREVILLE, J.F. [1977]: “Le développement de l'assurance est-il lié à celui de son environnement économique?", Vie et Science Economique, (juillet 1977), 34-39.

OUTREVILLE, J.F. [1978]: "The aggregation problem in the insurance demand equation: Review and investigation", Papier de recherche, Université de Pennsylvanie.

OUTREVILLE, J.F. [1979]: Un modèle Macroéconomique du Secteur de l'Assurance en France, Thèse d'Etat, Université d'Orléans.

POIRIER, D.J. [1976]: The Econometrics of Structural Change, North Holland, Amsterdam.

ROSA, J.J. [1977]: “La demande d'assurance non-vie: l'état actuel de la théorie”, Geneva Papers on Risk and Insurance, 5 (février 1977), 35-42.

SLOVIC, P. et al [1977]: "Preferences for insurance against probable small losses", Journal of Risk and Insurance, 44 (juin 1977), 237-258.

TAUBMAN, P. [1964]: A Synthesis of Saving Theory with Special References to the Components of Personal Saving, thèse de doctorat, Université de Pennsylvanie.

TAUBMAN, P. [1965]: “Permanent and transitory income effects", Review of Economics and Statistics, 47 (février 1965), 38-43.

YAARI, M. [1964]: “On the consumer's life time allocation process", International Economic Re. view, 5 (septembre 1964), 304-317.

YAARI, M. [1965]: “Uncertain life time, life insurance and the theory of the consumer", Review of Economic Studies, 32 (avril 1965), 137-150. 


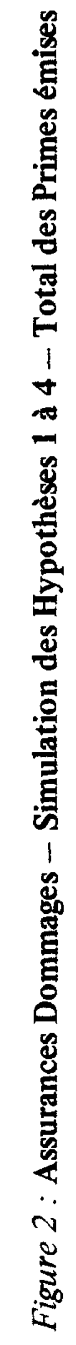




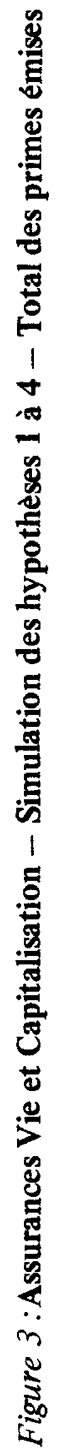




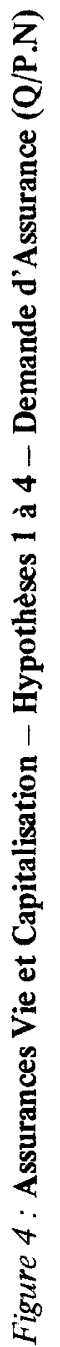

0
$\vdots$
$\sim$

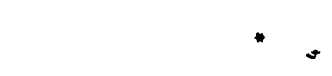


a

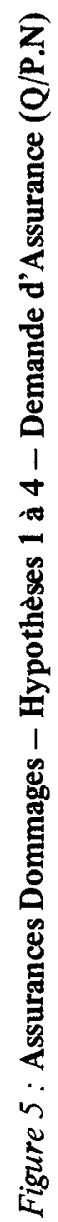

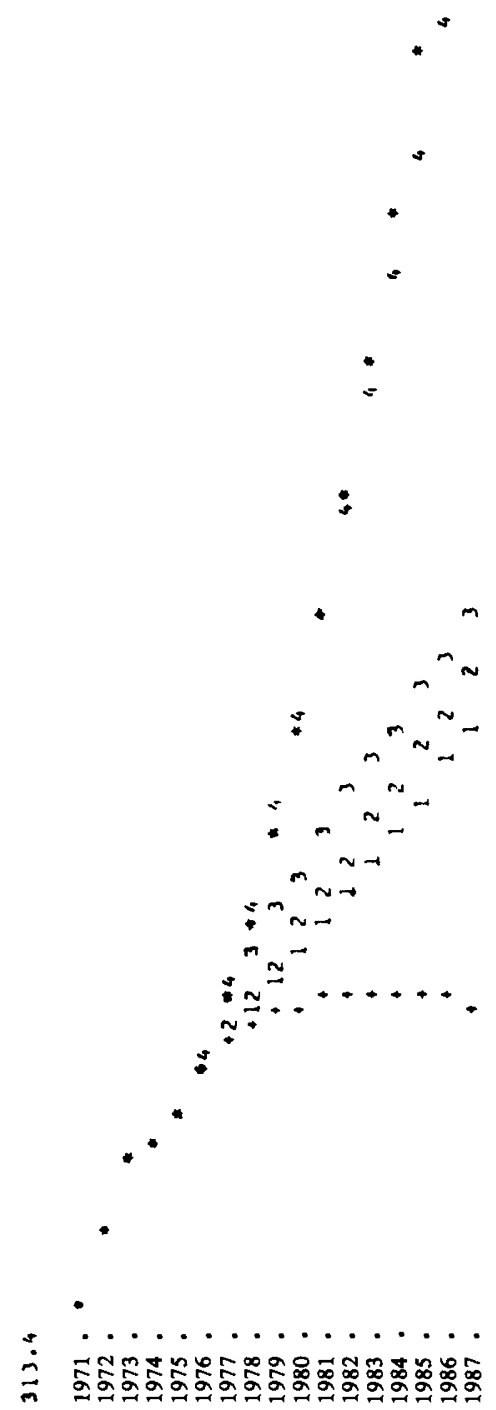

